\title{
Designing for Concreteness Fading in Primary Computing
}

\author{
Anthony Trory \\ University of Sussex \\ Brighton, England \\ a.trory@sussex.ac.uk
}

\author{
Kate Howland \\ University of Sussex \\ Brighton, England \\ k.1.howland@sussex.ac.uk
}

\author{
Judith Good \\ University of Sussex \\ Brighton, England \\ j.good@sussex.ac.uk
}

\begin{abstract}
In primary education, concepts are commonly introduced through concrete instantiations, such as physical manipulatives and kinaesthetic activities, with an expectation that learners will gradually move towards working with abstract representations. There has been considerable research in subjects such as mathematics on how children can move from working with concrete to abstract materials, but relatively little research on how this can be achieved in computing, which has recently become a more prominent subject at primary level. This paper reports on the design and evaluation of a low-fidelity prototype learning environment that aims to teach children aged 9-10 about a key computing concept (internet routing), using a concreteness fading approach commonly applied in mathematics. An empirical study with 59 children showed that those following a concreteness fading progression scored significantly higher on a post-test than those using a concrete only prototype, and had an increase in positive attitude towards computing in line with alternative approaches. We highlight the potential for an augmented reality implementation of the prototype to support investigation of further key questions raised by this research.
\end{abstract}

\section{Author Keywords}

Technology Enhanced Learning; Primary Education; Computing Education; Children; Grounded Cognition; Design Research

\section{ACM Classification Keywords}

- Human-centered computing Interface design prototyping

- Applied computing Interactive learning environments

- Social and professional topics $\sim \mathrm{K}-12$ education

\section{INTRODUCTION}

The introduction of a new computing curriculum in England [11] mirrors growing international interest in fostering younger children's learning in this area. The reaction of primary school teachers to the new curriculum has been mostly positive, however some have expressed concern that they do not have the resources to teach the more advanced areas of the curriculum [24]. Computing at School [9] have been developing and distributing new resources through the Barefoot [3] and Quickstart [25] schemes. A recurring theme is the recommendation of concrete learning activities; a practice already common in maths and science education, where it makes use of contextualisation, physical activity, and/or tangible manipulatives. A significant proportion of primary school teachers use this approach in their computing classes, with many believing that physicality itself is beneficial to learning [30].

Much of the inspiration for these activities comes from the CS Unplugged resources and design principles [10]. However, the primary aim of CS Unplugged is to increase children's interest in computer science, and there is currently little evidence on whether these activities promote the learning of transferable knowledge and skills. The computing curriculum in England requires that by age 11, all pupils "can understand and apply the fundamental principles and concepts of computer science, including abstraction, logic, algorithms and data representation" [11]. Introducing children to concepts in a concrete way may be more engaging, and necessary at younger ages, but how best to progress towards abstract understanding whilst maintaining motivation for the subject remains an open question.

One possibility is concreteness fading, an approach originating from Bruner's Modes of Representation [7]. Concreteness fading posits that the transition between concrete and abstract should be a directed progression through multiple external representations, with an initial grounded representation becoming more idealised over time. It has been suggested that the explicit linking of referents within distinct representations is important to the success of this approach [15], and technology enhanced learning environments could potentially provide good support for this, for example, through combining tangible manipulatives and augmented reality.

The study reported here forms part of a larger design-based research project investigating the ways in which digitally augmented tools might support progress from concrete to abstract in the learning of computing concepts for children aged 11 and under. The work presented here covers the design of a low-fidelity prototype learning environment, and an empirical study investigating whether the concreteness fading approach commonly used in mathematics education 
can be effectively applied to computer science learning in this context.

The objective of the empirical study was to quantitatively evaluate the concreteness fading approach, as applied in a prototype learning environment for teaching children aged 910 about the computing concept of internet routing. Two research questions were investigated within this context: 1) is concreteness fading an effective method of teaching an abstract computing concept to children aged 9-10, and 2) is there a relationship between concreteness fading and pupil attitudes towards computing?

\section{RELATED WORK}

The requirements for concrete activities that promote transferable knowledge are not yet fully understood, but relevant evidence is emerging from the field of grounded cognition: a paradigm incorporating theories of cognitive simulation, cognitive linguistics, and situated action [5]. Although there is no consensus on the extent to which cognition requires grounding, there is an increasing body of evidence that suggests conceptual processing often involves strong interaction with sensorimotor systems (see [20] for a review). Grounded cognition has promising applications in education, with studies showing that tangible manipulatives can benefit learning in English [16] and science [21]. There is some evidence to suggest that the learner may not actually need direct interaction with physical manipulatives, with research showing bodily gestures may influence cognitive simulation more than the actions they signify [17].

In fact, merely activating pre-existing sensorimotor experiences can be beneficial, as in the case of conceptual metaphor. It has been proposed that most abstract thought is grounded in metaphor, including mathematical concepts such as algebra and logic [22]. Although this may be considered a radical position, the use of metaphor in computing is explicit and pervasive, and fulfils both design and pedagogical roles [8]. A rich conceptual metaphor can provide an engaging experience and ground concepts in a way that supports constructivist learning. Computing skills are already often introduced to children in contextualised activities, such as debugging the process of making a pizza [4]. However, whereas a high-level metaphor such as algorithms are like recipes may seem innocuous, care must be taken to avoid creating misconceptions in the mind of the learner.

Poorly designed activities may allow the learner to complete tasks without developing an accurate conceptual understanding of their actions. Commenting on the use of manipulatives in mathematics, Ball notes that "[al]though kinaesthetic experience can enhance perception and thinking, understanding does not travel through the fingertips and up the arm" [2]. This caution must be considered not only in relation to kinaesthetic learning, but to all varieties of grounded learning. The learner's ability to create generalisations from the concrete, or to connect mutual referents between representations, cannot be taken for granted.

Several recent studies have investigated the benefits of concreteness within multiple external representation learning environments, using the concreteness fading approach. Goldstone and Son [18] developed a computer simulation to teach undergraduate students the scientific principle of competitive specialisation. The learning environment presented this concept using an ants and food metaphor, and comprised two interactive visual animations: the first using simple drawings of ants and fruit (concrete), and the second using dots and patches (abstract). In the study, Goldstone and Son controlled the order of presentation i.e. abstract only, concrete only, concreteness fading (concrete followed by abstract), and concreteness introduction (abstract followed by concrete), and found that concreteness fading led to the best performance on both near and far transfer tests. However, when the effectiveness of this simple two-stage visualisation approach to concreteness fading was investigated with younger children ( $M=12.7$ years) in the context of teaching the principles of electrical circuits, the earlier findings were not replicated.

An alternative to the two-stage process can be found in Bruner's modes of representation, perhaps more commonly known as the Concrete Pictorial Abstract approach. Bruner posited that knowledge could be understood in three distinct modes: enactive (environmental interaction), iconic (visualisation), and symbolic (language), and that following this sequence would provide an optimum learning progression. In 2014, Fyfe et al. published their 'Theoretical Model of Concreteness Fading' [15], in which the authors adopt Bruner's three-stage approach. Furthermore, they argue that the role of the iconic stage is to explicitly link features of the enactive and symbolic stages as mutual referents in the mind of the learner and, to do so most effectively, the iconic should introduce the symbolic within the context of the enactive. This form of simultaneous presentation can be found in several examples of related learning materials, but is not ubiquitous. Several studies in multimedia learning seem to support the authors' position, by demonstrating the importance of both spatial and temporal contiguity in the context of the split-attention principle [1].

Fyfe et al. applied their model to the design of learning materials to teach children (age $\mathrm{M}=8$ years) the principles of mathematical equivalence [14]. The enactive stage implemented a combination of metaphor (balance scales as an equation) and physical manipulation (placing objects on the scales to achieve balance). The symbolic stage used standard mathematical notation only, and the iconic stage showed a simple visualisation of the balance scales annotated with its mathematical referents. The materials were evaluated using the same experimental design described above, and it was found that concreteness fading led to the highest performance on the transfer test, followed by abstract 
only, concreteness introduction, and finally concrete only. Fyfe and colleagues then performed a further experiment to rule out the alternative interpretation that playful activity merely primes children for abstract learning. It was found that, within the concreteness fading sequence, conceptually relevant concreteness raised post-test performance more than conceptually irrelevant concreteness. The design principles set out in Fyfe et al.'s model were followed when developing the low fidelity prototype described in this paper.

Many technology-enhanced learning tools aimed at computing education for children have placed importance on concrete instantiations and/or sensorimotor experiences. Existing tools have most commonly focussed on supporting programming activities, rather than introducing wider computing concepts. The original Logo floor turtle has inspired many robotics-based approaches to bringing programming to the physical world including Curly Bot [13], Bee-Bots [31], Thymio [28], and the robots developed by Bers et al. as part of their robotics-based curriculum [6]. There are also many tangible programming tools in which code is composed through physical manipulation, including Electronic Blocks [33] and CHERP [32], and other forms of physical manipulatives such as Cricket [27] and Topobo [26]. Some tools aimed at younger children, such as Scratch Jr. [12] and Tynker [23] focus on screen-based interactions, but draw on children's everyday experiences of physical interaction, using interface metaphors such as the "puzzle piece" code composition popular across novice programming environments.

Although many tools make use of concrete elements to support computing learning for younger children, no existing tools provide explicit support for learners as they move from concrete to abstract representations. A tool that blends physical and onscreen interactions has potential to support such a move. Platforms such as Osmo [19] include both physical and onscreen elements, but require attention to be split between the screen and manipulatives. A full augmented reality learning environment could enable the annotation of tangible objects with iconic and symbolic information. The potential to manipulate the modality, appearance, and quantity of virtual representations presented to the learner at any time, would make it possible to create a gradual transition from concrete to abstract.

\section{PROTOTYPE DESIGN}

A low-fidelity prototype learning environment was created to explore the potential for augmented reality to support concreteness fading in introducing computing concepts. In the conceptual design stage of this study, various computing concepts were explored and evaluated for their appropriateness, drawing inspiration from CS Unplugged resources. A series of low-fidelity prototype learning environments were created based on the existing empirical and theoretical work on concreteness fading in mathematics, described previously. An initial prototype was designed for teaching children about sorting algorithms using balance scales but it was considered that the abstract representation was likely to be too advanced for the target age group. A second prototype was developed that introduced finite state machines using a pirate themed treasure hunt game, with the concrete representation fading into a more abstract diagram. In-school evaluation with 12 children showed that the concrete representation was highly engaging and motivating, but the metaphor did not help introduce the concept to children of this age range. In the third and final prototype, the treasure hunt activity was adapted to teach children about

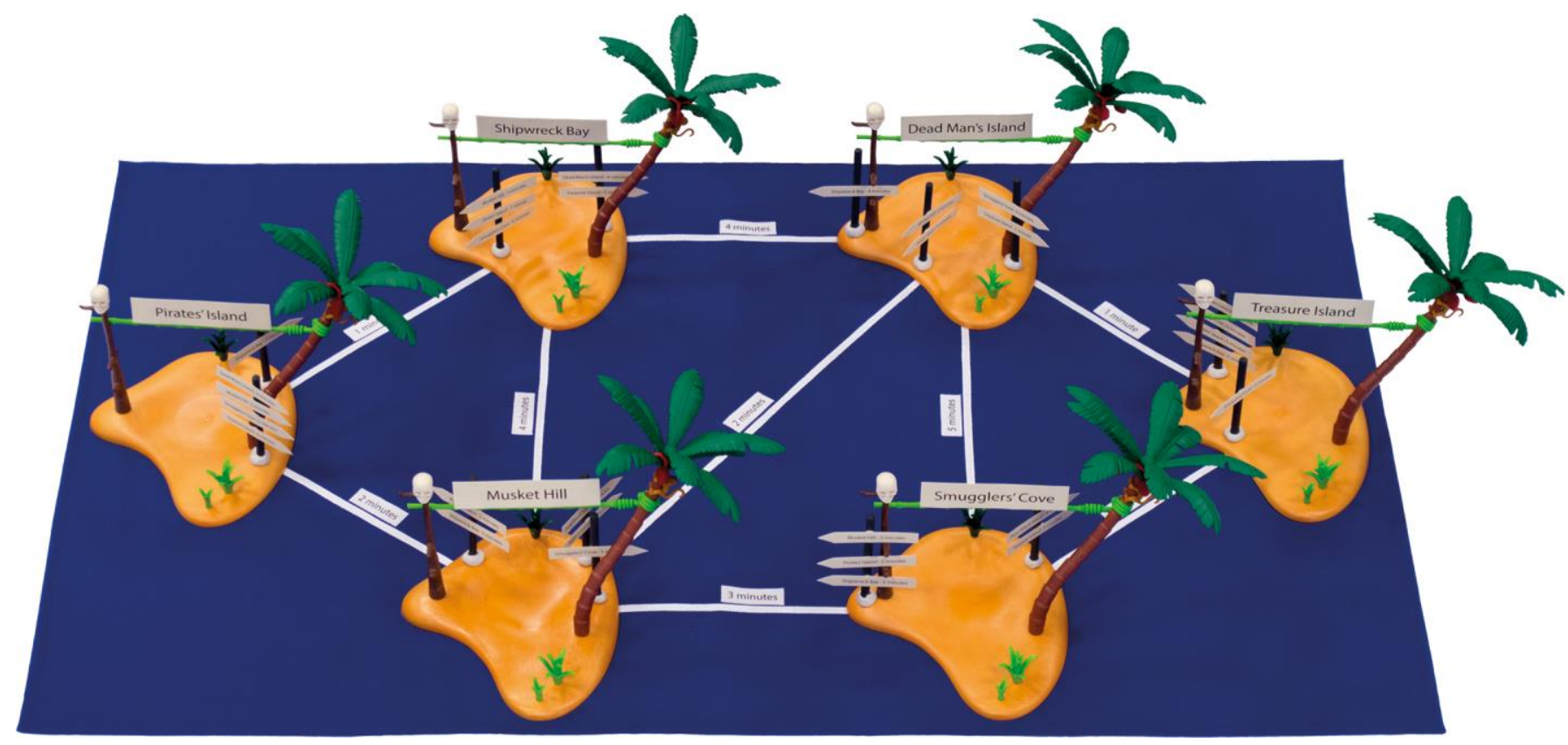

Figure 1. Pirate island concrete representation 


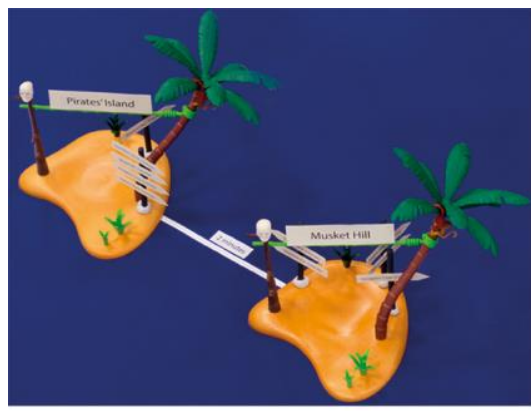

concrete

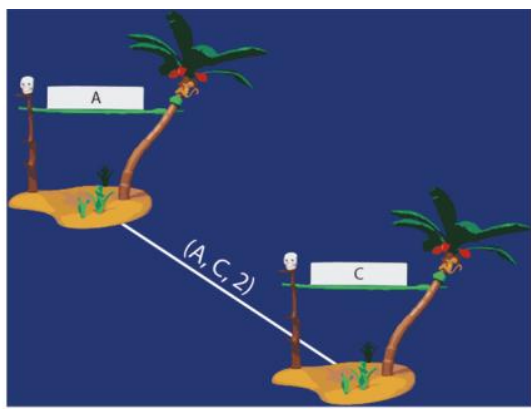

intermediate

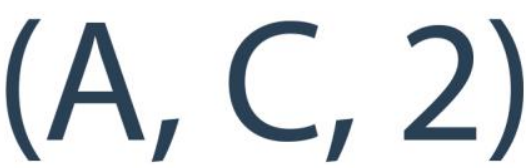

abstract

Figure 2. Fading representations of an edge

the fundamentals of network structure and internet routing tables. After in-school evaluation with three children, this prototype was selected to be used in the study. The children who took part in the prototype evaluations varied in terms of their ability and attitude towards computing, as judged and selected by the school.

\section{Prototype Implementation}

The prototype was designed to support movement between concrete, intermediate, and abstract representations. In an augmented reality version of the tool, the move between representations would take place on screen. In this prototype, the researcher presented the different representations to participants by hand.

The concrete stage of the prototype employs the metaphor of a group of islands, with each inhabited by a pirate that wishes to communicate with their friends. Messages may only be sent between islands using carrier parrots. A parrot may only fly along specified flight paths, and must follow direction signs to ensure the quickest route between source and destination. In this scenario, islands represent the nodes of a network, flight paths represent the edges of a network, parrots represent data packets, and a direction sign represents an entry in a routing table. The concrete representation was created using six Playmobil pirate islands and a $100 \mathrm{~cm} \mathrm{x}$ $60 \mathrm{~cm}$ sheet of thick blue cotton (shown in Figure 1).
Flight paths were added and labelled with time e.g. "2 minutes" (the stated time was unrelated to the length of the flight path). The prototype does not change state as the user interacts with it, but acts as a three-dimensional visual representation. The parrot (not shown) was moved between islands by hand, acting as a token that enabled users to keep track of their current position within a sequence of actions, and to focus their attention on the relevant spatial area.

The abstract representation of the network structure was an 'edge list': a list of all unique connections between nodes. The abstract representation of the internet routing tables was a collection of data tables, one per node. Both concepts were presented side-by-side on a single sheet of A4 paper. All context and extraneous perceptual detail was removed, for example nodes were labelled with letters and cost was an unspecified unit.

The intermediate representation attempts to explicitly link features of the concrete and abstract stages as mutual referents. To link flight paths with edges, a simplified pictorial map of the islands was created and then annotated with the relevant abstract features, as shown in Figure 2. To link the direction signs with the routing tables, the direction signs were removed from the context of the islands and placed into tabular form, as shown in Figure 3. Again, both concepts were shown side-by-side on a single sheet of A4 paper.

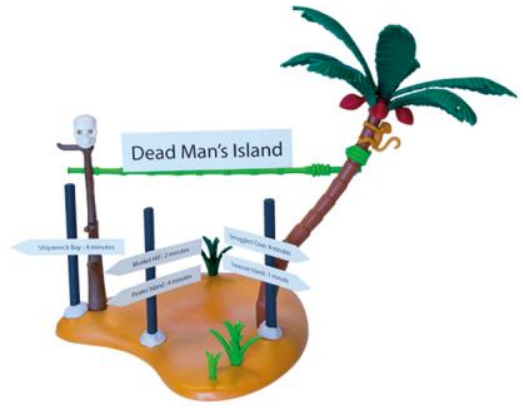

concrete

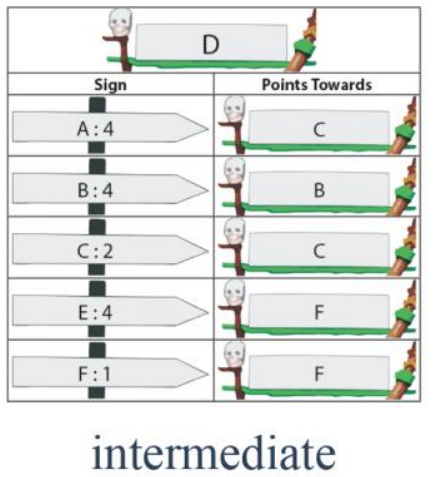

\begin{tabular}{|c|c|c|}
\hline \multicolumn{3}{|c|}{ D } \\
\hline Destination & Cost & Next Node \\
\hline A & 4 & C \\
\hline B & 4 & B \\
\hline C & 2 & C \\
\hline E & 4 & F \\
\hline F & 1 & F \\
\hline \multicolumn{3}{|c}{ abstract }
\end{tabular}

Figure 3. Fading representations of a routing table 


\section{Narration Scripts}

The learner was guided through the activity by narration, which in this prototype was provided by the researcher reading a script. In the intermediate stage, the script was almost identical to that of the abstract stage, but some phrasing was altered to reflect the different presentation.

In the following script excerpt from the concrete stage, the researcher explains how to use routing tables to find the lowest cost route between two nodes:

"If the parrot wants to deliver a message from Musket Hill to Shipwreck Bay, it first looks at the direction signs on Musket Hill and finds the one with Shipwreck Bay written on it. It can see that the time needed to fly to Shipwreck Bay is 3 minutes, and it knows this is the fastest route to its destination. The parrot then sees that the direction sign is pointing towards Pirates' Island, so this is the island it must fly to next. When the parrot arrives at Pirates' Island it repeats the process: it first finds the direction sign with Shipwreck Bay written on it, sees it pointing towards Shipwreck Bay and then flies to it."

In the following script excerpt from the intermediate stage, the researcher again explains how to use routing tables to find the lowest cost route between two nodes, but using more abstract terminology:

"If we want to travel from node $C$ to node $B$, we first find node $C$ 's routing table and search the destination column for the row with node $B$ written in it. We can see that the cost of travelling to node B is 3, and we know this is the lowest cost route to our destination. We then look in the 'next node' column and see node A, so this is the node we must travel to next. When we arrive at node $A$ 's routing table we repeat the process: we first find the row with node $B$ written in it, see that node $B$ is the next node to travel to, then we travel to it."

\section{METHOD}

\section{Design}

This study used a between-groups design, with condition as the independent variable. Four groups were compared: abstract (A), concrete (C), concreteness fading (CF), and concreteness introduction $(\mathrm{CI})$, with each differing only in the conceptual representation used in the prototype with which they interacted. All participants progressed through three learning stages, as shown in Table 1. The first dependent variable, intended to measure instructional efficacy, was the difference between pre- and post-test scores. The second dependent variable, intended to measure attitudinal change, was the difference in pre- and post-survey scores. Participants' mean scores from a recent Cognitive Abilities Test (CAT) were recorded as a participant variable and used to implement matched random assignment to groups (the $\mathrm{CAT}^{1}$ is a test used in the UK to provide a standardised measure of cognitive reasoning ability that is normally distributed around 100).

$\begin{array}{rccc} & \text { Stage 1 } & \text { Stage 2 } & \text { Stage 3 } \\ \text { A } & \text { Abstract } & \text { Abstract } & \text { Abstract } \\ \text { C } & \text { Concrete } & \text { Concrete } & \text { Concrete } \\ \text { CF } & \text { Concrete } & \text { Intermediate } & \text { Abstract } \\ \text { CI } & \text { Abstract } & \text { Intermediate } & \text { Concrete }\end{array}$

Table 1: Study conditions

\section{Participants and Settings}

The sessions took place in a local primary school, during normal hours. The pre-test and pre-survey were completed in the participants' classroom. The learning activities, post-test, and post-survey were completed in a nearby corridor workspace. All pupils in year five of the school were invited to participate: a total of 95 pupils. Those who had previously contributed to the evaluation of learning materials were excluded from the sample. The final sample size was 59, comprising 30 girls and 29 boys, aged between nine and ten years $(M=10.27$ years, $S D=0.27)$, with a mean CAT score of $106.37\left(\mathrm{SD}=12.16, \mathrm{X}_{(1)}=80, \mathrm{X}_{(\mathrm{n})}=135\right)$. Participants were rank ordered on CAT score and then assigned to conditions using blocked randomisation. The groups were then manually adjusted to achieve gender balance by exchanging participants with those of opposite genders but closely matched CAT scores.

$\begin{array}{rcccc} & \text { A } & \text { C } & \text { CF } & \text { CI } \\ \mathbf{n} & 15 & 14 & 15 & 15 \\ \text { Males } & 8 & 7 & 7 & 7 \\ \text { Females } & 7 & 7 & 8 & 8 \\ \text { CAT M } & 105.53 & 106.29 & 106.67 & 107.00 \\ \text { CAT SD } & 11.54 & 13.34 & 12.16 & 12.83\end{array}$

Table 2: Group characteristics

\section{Materials}

\section{Conceptual Representations and Worksheets}

In the design stage of this study, a simple network map was created, consisting of six nodes and nine edges, with costs ranging from one to five. This map was then distorted to create two further, superficially different, network maps. All three maps were then translated into the conceptual

\footnotetext{
${ }^{1}$ https://www.gl-assessment.co.uk/products/cognitiveabilities-test-cat4/
} 
representations required for the study, as shown in Table 1. All participants were introduced to network one, followed by network two, and finally network three, regardless of the condition to which they were assigned.

For each network, a short worksheet comprising two activities was created. Activity one specified a route across the network and required the participant to find the total route cost by summing the individual cost of each edge traversed. Activity two specified a source and destination node and required the participant to find each of the nodes visited along the route shown in the routing tables. Both questions were separated into a part $\mathrm{A}$ and a part $\mathrm{B}$, with part $\mathrm{A}$ being completed and narrated by the researcher, and part $\mathrm{B}$ being completed by the participant alone.

\section{Attainment Test and Attitudinal Survey}

The attainment test comprised three printed sheets: an introductory page, a six-question multiple-choice worksheet, and a conceptual representation of a nine-node network and its associated routing tables. As in the learning stages, an initial network map was designed and then distorted to produce a second superficially different map. From these maps, two editions of the attainment test were developed: A and B. Approximately half of the participants received edition $\mathrm{A}$ as the pre-test and edition $\mathrm{B}$ as the post-test, while the remainder received edition $\mathrm{B}$ as the pre-test and edition A as the post-test.

Although visually similar to the abstract representation used in the learning stage, in this case the network was contextualised as a fictional example of internet structure in the USA, and therefore could be considered a test of near transfer. Edges were referred to as 'data lines' and nodes referred to as 'routers', with individual routers named after cities, for example 'New York', 'Denver', and 'San Francisco'. The introductory page gave brief definitions of each of the new terms, for example: "The internet is a global network of computers that can send data to each other. The connections between these computers are called data lines".

The multiple-choice attainment test comprised six questions, each with four potential answers and a fifth option of "not sure". It was intended that each question should be scored at one point for a correct answer, and zero points for all other options. However, one answer option under question three was scored a half point due to presentational error.

The first three questions related to edge lists, for example, the question "how many routers can Oklahoma City send data to directly?" required participants to understand that edges represent connections between nodes. To answer the question correctly, the participant had to scan the edge list and find the total number of edges containing the specified node. One alternative, but incorrect, method of answering this question would have been to instead refer to the routing tables and count the number of times the destination and next node column contained the same router name. The remaining two questions in this section were: "how long does it take for data to travel between Phoenix and Houston directly?" and "if data is sent from San Francisco to Boston using these data lines $[\ldots]$, how long would it take the data to travel from Salt Lake City to Miami?"

The final three questions related to routing tables, for example, the question "what is the fastest that data could be sent from Boston to Miami?" required participants to understand that routing tables supersede edges when finding the lowest cost route. To answer the question correctly, the participant had to find the table belonging to the first node, then the row referring to the second node, and then the value in the next node column. One alternative, but incorrect, method of answering this question would have been to instead refer to the edge list, and find the cost of the edge that connected the two nodes directly. The remaining two questions in this section were: "if data is sent along the fastest route from Oklahoma City to Salt Lake City, which router will it be sent to first?" and "the fastest route from Portland to Houston is $[\ldots]$, which routers are missing from this route?"

The attitudinal survey comprised the following five statements: 1) I like computing, 2) I am good at computing, 3) I like the challenge of computing, 4) computing is fun, and 5) I want to find out more about computing. These statements were selected, based on their relevance, from those previously used to assess the impact of a game making project on attitudes towards computing [29]. Responses were scored on a five point Likert scale, presented as a series of faces, progressing from sad to happy.

\section{Procedure}

The study ran from April to June 2017 and was conducted with each of the three classes consecutively. Each participant completed two sessions: the collectively administered presurvey and pre-test session (S1), and later, the individually administered treatment, post-survey, and post-test session (S2). Block randomization was used to ensure that the duration between $\mathrm{S} 1$ and $\mathrm{S} 2$ was matched across groups, resulting in a mean of 17.61 days $\left(\mathrm{SD}=8.23, \mathrm{X}_{(1)}=8, \mathrm{X}_{(\mathrm{n})}=\right.$ 33).

The S1 sessions lasted approximately 15 minutes. After introduction, the pre-survey was distributed to the participants, completed, and collected. The pre-test was then distributed to the participants, face-down and alternating edition $A$ and edition $B$ such that no participant was seated next to another with the same edition of the pre-test. Participants were asked to turn over their introductory page while the researcher read aloud from it. Participants were then asked to turn over the remaining pages and given seven minutes to complete the multiple-choice worksheet. Finally, participants were asked to put their pencils down, and the pre-tests were collected.

The S2 sessions lasted approximately 35 minutes. Participants were first given an overview of the session and asked for verbal assent to continue, with all agreeing to do 
so. The procedure for each of the three learning stages was the same. The researcher began by revealing the conceptual representation to the participant and then introduced edges by narration while pointing at the key features. Next, the researcher revealed the worksheet and completed activity A while narrating their actions, with participants then asked to complete activity B alone. This two stage process was then repeated to introduce routing tables. It was planned that if a participant were to query a point during narration, or provide an incorrect answer to an activity, the researcher would repeat the relevant portion of the script a maximum of two times before moving on. However, no participant needed more than one repetition. After the learning stages were finished, the post-survey and post-test were completed as in the procedure for $\mathrm{S} 1$ described above.

\section{RESULTS}

\section{Attainment Test}

The overall mean pre-test score was 1.26 out of 6 (SD = 1.13), and the overall mean post-test score was 2.37 out of 6 $(\mathrm{SD}=1.18)$. Independent-samples t-tests were run to determine if gender differences existed in pre-test or posttest scores. On the pre-test, females $(\mathrm{M}=1.40, \mathrm{SD}=1.15)$ achieved higher scores than males $(\mathrm{M}=1.12, \mathrm{SD}=1.12)$, but the difference was not statistically significant $(\mathrm{M}=0.28$, $95 \%$ CI $[-0.31,0.87], \mathrm{t}(57)=0.95, \mathrm{p}=.347)$. On the posttest, males $(M=2.48, S D=1.07)$ achieved higher scores than females $(\mathrm{M}=2.27, \mathrm{SD}=1.29)$, but the difference was not statistically significant $(\mathrm{M}=0.22,95 \% \mathrm{CI}[-0.40,0.83], \mathrm{t}(57)$ $=0.70, \mathrm{p}=.487$ ).

Linear regression was used to confirm the relationship between the planned covariates and the dependent variable. It was established that CAT score could statistically significantly predict post-test score, $\mathrm{F}(1,57)=11.53, \mathrm{p}=$ .001 , with an adjusted $\mathrm{R} 2=15.4 \%$, and that pre-test score could statistically significantly predict post-test score, $\mathrm{F}(1,57)=12.63, \mathrm{p}=.001$, with an adjusted $\mathrm{R} 2=16.7 \%$. Linear regression also showed that CAT score did not statistically significantly predict pre-test score, $\mathrm{F}(1,57)=$ $2.45, \mathrm{p}=.123$, with an adjusted $\mathrm{R} 2=2.4 \%$.

An ANCOVA was run to determine the effect of conceptual representation on post-test scores after controlling for pretest and CAT scores. The adjusted means per condition were as follows: concrete $\mathrm{M}=1.83, \mathrm{SE}=0.26$; concreteness introduction $\mathrm{M}=2.32, \mathrm{SE}=0.25$; abstract $\mathrm{M}=2.44, \mathrm{SE}=$ 0.25 ; concreteness fading $\mathrm{M}=2.87, \mathrm{SE}=0.25$. After adjustment, there was a statistically significant difference in post-test scores between groups, $\mathrm{F}(3,53)=2.79, \mathrm{p}=.049$, partial $\eta 2=.14$. Planned contrasts were run, with the concreteness fading condition as reference. The concreteness fading condition led to statistically significantly higher posttest scores than the concrete condition (Mdiff $=1.04$, $\mathrm{CI}[0.31,1.77], \mathrm{p}=.006)$. However, the concreteness fading condition did not lead to statistically significantly higher post-test scores than the concreteness introduction condition (Mdiff $=0.55, \mathrm{CI}[-0.16,1.26], \mathrm{p}=.127$ ) or the abstract condition (Mdiff $=0.42, \mathrm{CI}[-0.29,1.16], \mathrm{p}=.239$ ). The adjusted learning gains are shown in Figure 4, and broken down by gender in Figure 5.

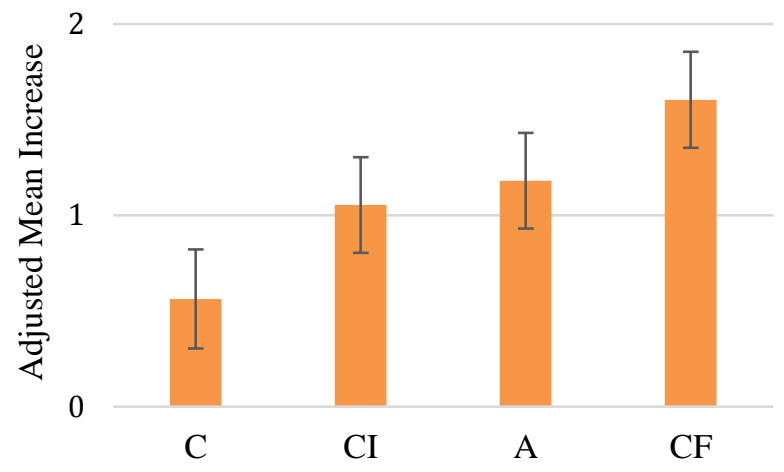

Figure 4. Learning gains. Derived from ANCOVA adjusted means, with covariates evaluated at the following values: CAT $=106.37$ and pre-test $=1.26$. Error bars represent standard error.

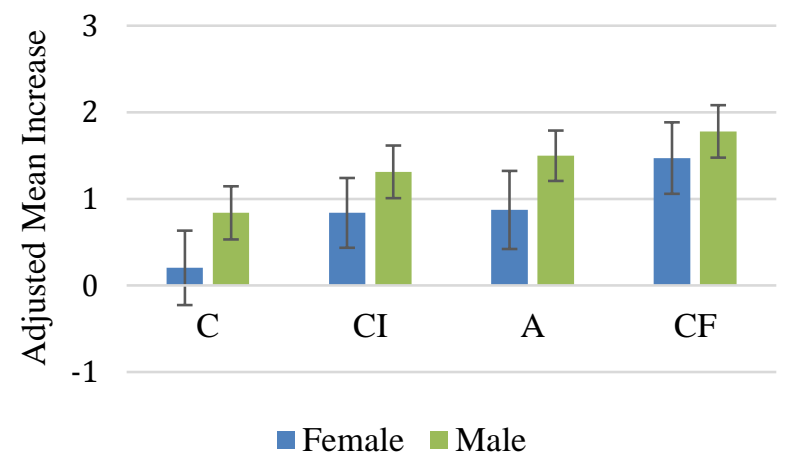

Figure 5. Learning gains, by gender. Derived from ANCOVA adjusted means, with covariates evaluated at the following values: $($ females) $\mathrm{CAT}=107.13$ and pre-test $=1.40$; (males) CAT $=105.59$ and pre-test $=1.12$. Error bars represent standard error.

\section{Attitudinal Survey}

Analysis of the survey responses was conducted on the sum of the five statements, creating a single attitudinal measure on a 25 point scale and enabling the use of parametric tests. The overall mean pre-survey score was 20.12 out of 25 (SD $=4.39$ ), and the overall mean post-survey score was 21.80 out of $25(\mathrm{SD}=3.17)$. Independent-samples t-tests were run to determine if gender differences existed in pre-survey or post-survey scores. On the pre-survey, males ( $\mathrm{M}=20.90, \mathrm{SD}$ $=4.45)$ had higher scores than females $(\mathrm{M}=19.37, \mathrm{SD}=$ 4.26), but the difference was not statistically significant (M $=1.53,95 \%$ CI $[-0.74,3.80], \mathrm{t}(57)=1.35, \mathrm{p}=.183)$. On the post-survey, males $(\mathrm{M}=22.66, \mathrm{SD}=2.37)$ again had higher 
scores than females $(M=20.97, S D=3.64)$, and the difference was statistically significant $(\mathrm{M}=1.69,95 \% \mathrm{CI}$ $[0.81,3.30], \mathrm{t}(57)=2.104, \mathrm{p}=.040)$.

Linear regression was used to confirm the relationship between the planned covariate and the dependent variable. It was established that pre-survey score could statistically significantly predict post-survey score, $\mathrm{F}(1,57)=39.78$, $\mathrm{p}<$ .001 , with an adjusted $\mathrm{R} 2=40.1 \%$.

An ANCOVA was run to determine the effect of conceptual representation on post-survey scores after controlling for pre-survey scores. The adjusted means per condition were as follows: concrete $\mathrm{M}=21.03, \mathrm{SE}=0.65$; abstract $\mathrm{M}=21.67$, $\mathrm{SE}=0.63$; concreteness introduction $\mathrm{M}=21.72, \mathrm{SE}=0.63$; concreteness fading $\mathrm{M}=22.72, \mathrm{SE}=0.63$. After adjustment, there was not a statistically significant difference in postsurvey scores between groups, $\mathrm{F}(3,54)=1.19, \mathrm{p}=.322$, partial $\eta 2=.06$. The adjusted attitudinal improvements are shown in Figure 6, and broken down by gender in Figure 7.

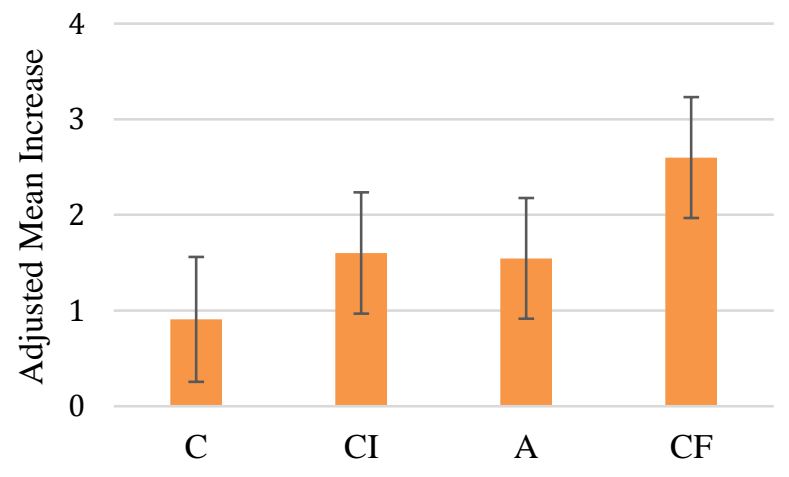

Figure 6 Attitudinal improvement. Derived from ANCOVA adjusted means, with covariates evaluated at the following values: pre-survey $=20.12$. Error bars represent standard error.

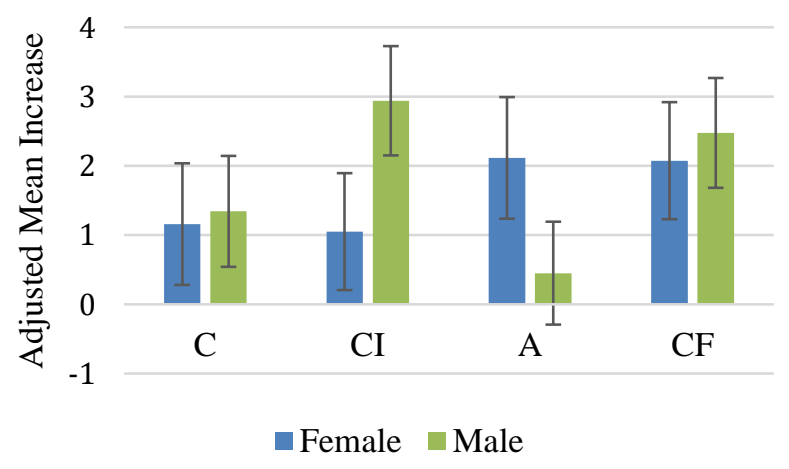

Figure 7 Attitudinal improvement, by gender. Derived from ANCOVA adjusted means, with covariates evaluated at the following values: (females) pre-survey $=19.37$; (males) presurvey $=20.90$. Error bars represent standard error.

\section{DISCUSSION}

Conceptual representation was shown to make a significant difference to post-test scores, when controlling for pre-test and CAT scores. The concreteness fading version of the prototype resulted in higher post-test scores than the other versions, a difference that was statistically significant between the concrete condition and the concreteness fading condition. The ranking of the post-test means across different conditions (concrete, concreteness introduction, abstract, concreteness fading) was in line with the findings of Fyfe et al. [14] who investigated the approach in mathematics learning with pupils of the same age. When examining the learning gain by gender, the benefits of concreteness fading appear to be more pronounced for females than males.

With respect to the relationship between concreteness fading and pupil attitudes towards computing, there was a nonsignificant trend for the attitudinal post-survey scores to be highest for the concreteness fading version of the prototype. However, the ranking of different conditions on attitudinal gain altered when broken down by gender. Although the trend is in favour of concreteness fading overall, it ranks second for both males and females when considered separately, with a trend for greater attitudinal increase for males with concreteness introduction, and a very slight trend in favour of the abstract only condition for females.

The findings suggest that concreteness fading, as implemented in this prototype, is a promising approach for introducing computing concepts such as internet routing to children aged 9-10, and warrants further investigation. In particular, there are significant benefits over the introduction of such concepts through concrete only materials.

There are some limitations to the evaluation of the lowfidelity prototype described in this paper. Firstly, the evaluation focussed on one computational concept only: that of internet routing. Further work is necessary to determine the extent to which the concreteness fading approach is more broadly applicable to learning computing. Furthermore, evaluation of pupil learning focused on near transfer only, i.e. the extent to which children could apply the concepts they had learnt in analogous situations. Again, further work is needed to investigate whether far transfer is possible. Finally, a sample size of 59, although appropriate for an exploratory study of this nature, does not allow conclusive statements about the efficacy of concreteness fading to be made. Further work should aim to increase the sample size.

Opportunities and research questions for digitally augmented tools to support concreteness fading

The prototype evaluated in this paper represents a first step in investigating concreteness fading approaches to computing education at primary level. The low-fidelity prototype was appropriate in the context of an empirical study: as noted above, it was important to determine whether the approach might be of benefit before beginning to implement digital prototypes. However, this prototype required extensive setup, and the transition between stages 
had to be carried out manually by the researcher. In addition, all the bespoke materials relate to one concept and learning activity only.

The next stage is to develop a digitally augmented version of the concreteness fading prototype. Such a solution could provide support for researchers (and eventually, teachers) in managing the activity, and would allow a variety of different concepts to be taught using a series of different metaphors. There are a range of technologies that could be used to implement this, including augmented reality, and potentially other forms of tangible user interfaces and tabletop touch screens. However, any digital tool would need to run on equipment readily available in classrooms. For example, an augmented reality application that runs on the tablets most commonly found in primary school classrooms may be a viable option. A further advantage of a digitally augmented environment is the potential to investigate the benefits of making the "fading" more gradual. In the current, nontechnology instantiations of concreteness fading, fading is achieved by moving between distinct setups and representations at each stage. For example, in the setup described in this paper, children must move from a concrete, physical representation of the situation, to an intermediate representation which makes use of the physical aspects of the concrete situation (islands), but replaces informational elements (e.g. island names) with abstract elements (e.g. A). Children eventually move to a purely abstract representation with no physical elements. At each stage, they must transfer their knowledge of one representation onto the next, determining which elements of the representation map onto the elements of the next. This places a reasonably high cognitive load on the child, and it may be that some of the difficulties associated with moving from one representation to the next may in fact be due, not to a lack of understanding, but to an inability to make the correct mappings between the equivalent elements of the different representations.

In essence, concreteness fading is a stepwise process, with "fading" occurring at each change in representation. In a digitally augmented environment, concreteness fading could be implemented in a much more gradual, and fluid, manner. For example, the same base representation could be used throughout: starting with concrete instantiations of the elements in the representation, more abstract equivalents could gradually be overlaid onto the representation, slowly replacing the concrete elements with their more abstract counterparts. This would reduce the cognitive overhead inherent in switching between representations, and might allow the child to see more clearly the relationship between gradually more abstract versions of the same element.

Furthermore, implementing this as an adaptive system would have other potential benefits that should be investigated. For example, even if it is established that concreteness fading is a beneficial approach to learning computing concepts, it does not follow that the approach can simply be applied across the board for all children. Some children may need to spend longer at various stages, or even return to previous stages to consolidate their knowledge. An adaptive system could determine the points at which to gradually introduce concreteness fading, and at what pace.

Finally, it is not clear from existing research on concreteness fading whether concrete necessarily implies physical, or whether merely activating pre-existing sensorimotor experiences through conceptual metaphor is sufficient. Addressing this question for computing was beyond the scope of this study, but should be investigated further in future research.

\section{CONCLUSION}

The design and evaluation of the low-fidelity prototype presented in this paper has demonstrated that concreteness fading can be effectively used to teach computing concepts to children aged 9-10. This work is the first step in investigating the potential benefits of applying this approach in the design of learning environments for primary computing education.

The next stage in the research involves the implementation of a digitally augmented tool which will allow further investigation of the potential benefits. Augmented reality is a promising technology platform, as it avoids the need for split attention, and allows further investigation of a number of key questions, including the potential for more gradual fading, and the extent to which concrete representations need to be physical.

\section{SELECTION AND PARTICIPATION OF CHILDREN}

Ethical approval for this study was obtained from the University of Sussex Sciences \& Technology Cross-Schools Research Ethics Committee (SCITEC C-REC). All pupils in year five (95 children, aged 9-10) of a local primary school were invited to participate. The informed consent procedure involved written parental permission for all participants to take part in the tests and learning activities, and giving researchers access to the CAT scores. All child participants also gave verbal assent in response to an assent script prior to taking part in research activities. Class teachers put forward 15 children to take part in piloting the materials. The final sample size $(\mathrm{N}=59)$ excluded these 15 , and comprised 30 girls and 29 boys (all remaining pupils for whom we had obtained informed consent).

\section{ACKNOWLEDGEMENTS}

Many thanks to the teachers and children who donated their time and effort to our work. This study is part of doctoral research funded by the Engineering and Physical Sciences Research Council (EPSRC).

\section{REFERENCES}

1. Paul Ayres and John Sweller. 2014. The splitattention principle in multimedia learning. In The Cambridge Handbook of Multimedia Learning (2nd ed.), Richard E. Mayer (ed.). Cambridge University Press, 206-226.

2. Deborah Loewenberg Ball. 1992. Magical hopes: 
Manipulatives and the reform of math education. American Educator: The professional journal of the American Federation of Teachers 16, 2.

3. Barefoot Computing. About Barefoot. Retrieved January $19, \quad 2018 \quad$ from https://barefootcas.org.uk/about-barefoot/

4. Barefoot Computing. KS1 Pizza Pickle Activity. Retrieved January 19, 2018 from https://barefootcas.org.uk/wpcontent/uploads/2014/10/KS1-Pizza-PickleDebugging-Activity.pdf

5. Lawrence W. Barsalou. 2008. Grounded cognition. Annual review of psychology 59: 617-45.

6. Marina Umaschi Bers, Louise Flannery, Elizabeth R. Kazakoff, and Amanda Sullivan. 2014. Computational thinking and tinkering: Exploration of an early childhood robotics curriculum. Computers and Education 72: 145-157.

7. Jerome S. Bruner. 1966. Toward a theory of instruction. Harvard University Press, Cambridge, MA.

8. Timothy Colburn and Gary Shute. 2008. Metaphor in computer science. Journal of Applied Logic 6, 4: 526-533.

9. Tom Crick and Sue Sentance. 2011. Computing at school: stimulating computing education in the UK. In Proceedings of the 11th Koli Calling International Conference on Computing Education Research, 122-123.

10. CS Unplugged. Principles. Retrieved January 19, 2018 from http://csunplugged.org/principles/

11. Department for Education. 2013. National curriculum in England: Computing programmes of study. Retrieved January 19, 2018 from https://www.gov.uk/government/publications/natio nal-curriculum-in-england-computing-programmesof-study/national-curriculum-in-englandcomputing-programmes-of-study

12. Louise P. Flannery, Brian Silverman, Elizabeth R. Kazakoff, Marina Umaschi Bers, Paula Bontá, and Mitchel Resnick. 2013. Designing ScratchJr: Support for early childhood learning through computer programming. In Proceedings of the 12th International Conference on Interaction Design and Children, 1-10.

13. Phil Frei, Victor Su, Bakhtiar Mikhak, and Hiroshi Ishii. 2000. Curlybot: Designing a new class of computational toys. In Proceedings of the SIGCHI conference on Human factors in computing systems, 129-136.

14. Emily R. Fyfe, Nicole M. McNeil, and Stephanie
Borjas. 2015. Benefits of "concreteness fading" for children's mathematics understanding. Learning and Instruction 35: 104-120.

15. Emily R. Fyfe, Nicole M. McNeil, Ji Y. Son, and Robert L. Goldstone. 2014. Concreteness fading in mathematics and science instruction: A systematic Review. Educational Psychology Review 26, 1: 925.

16. Arthur M. Glenberg, Tiana Gutierrez, Joel R. Levin, Sandra Japuntich, and Michael P. Kaschak. 2004. Activity and imagined activity can enhance young children's reading comprehension. Journal of Educational Psychology 96, 3: 424-436.

17. Susan Goldin-Meadow and Sian L Beilock. 2010. Action's influence on thought: The case of gesture. Perspectives on psychological science 5, 6: 664-74.

18. Robert L. Goldstone and Ji Y. Son. 2005. The transfer of scientific principles using concrete and idealized simulations. Journal of the Learning Sciences 14, 1: 69-110.

19. Felix Hu, Ariel Zekelman, Michael Horn, and Frances Judd. 2015. Strawbies: Explorations in tangible programming. In Proceedings of the 14th International Conference on Interaction Design and Children, 410-413.

20. Markus Kiefer and Friedemann Pulvermüller. 2012. Conceptual representations in mind and brain: Theoretical developments, current evidence and future directions. Cortex 48, 7: 805-825.

21. Carly Kontra, Daniel J. Lyons, Susan M. Fischer, and Sian L. Beilock. 2015. Physical experience enhances science learning. Psychological science 26, 6: 737-749.

22. George Lakoff and Rafael E. Núñez. 2000. Where mathematics comes from: How the embodied mind brings mathematics into being. Basic Books, New York.

23. Neuron Fuel. Tynker. Retrieved January 19, 2018 from https://www.tynker.com

24. Pye Tait Consulting. 2017. After the reboot: The state of computing education in UK schools and colleges - teacher survey report. Retrieved from https://royalsociety.org/ /media/policy/projects/co mputing-education/pye-tait-teacher-surveyreport.pdf

25. Quickstart Computing. Introduction. Retrieved January $19, \quad 2018 \quad$ from http://primary.quickstartcomputing.org/introduction .html

26. Hayes Solos Raffle, Amanda J Parkes, and Hiroshi Ishii. 2004. Topobo: A constructive assembly system 
with kinetic memory. In Proceedings of the SIGCHI conference on Human factors in computing systems., 647-654.

27. Mitchel Resnick, Fred Martin, Robert Berg, Rick Borovoy, Vanessa Colella, Kwin Kramer, and Brian Silverman. 1998. Digital manipulatives: new toys to think with. In Proceedings of the SIGCHI conference on Human factors in computing systems, 281-287.

28. Fanny Riedo, Morgane Chevalier, Stéphane Magnenat, and Francesco Mondada. 2013. Thymio II, a robot that grows wiser with children. In Proceedings of IEEE Workshop on Advanced Robotics and its Social Impacts, ARSO. https://doi.org/10.1109/ARSO.2013.6705527

29. Judy Robertson. 2013. The influence of a gamemaking project on male and female learners' attitudes to computing. Computer Science Education 23, 1: 58-83.
30. Sue Sentance and Andrew Csizmadia. 2017. Computing in the curriculum: Challenges and strategies from a teacher's perspective. Education and Information Technologies 22, 2: 469-495.

31. Terrapin Software. Bee-Bot. Retrieved January 19, 2018 from https://www.bee-bot.us/

32. TK Tobotics Network. CHERP. Retrieved January 19 , 2018 from http://tkroboticsnetwork.ning.com/page/kiwisoftware-cherp

33. Peta Wyeth and Helen C. Purchase. 2003. Using developmental theories to inform the design of technology for children. In Proceeding of the 2003 conference on Interaction design and children, 93100. 